\title{
Modelagem Matemática e uma Proposta de Trajetória Hipotética
}

\author{
de Aprendizagem
}

\author{
Mathematical Modeling and a Proposal of Hypothetical Learning \\ Trajectory
}

\author{
Pamela Emanueli Alves Ferreira* \\ ORCID iD 0000-0002-9420-8536 \\ Karina Alessandra Pessoa da Silva ${ }^{* *}$ \\ ORCID iD 0000-0002-1766-137X
}

\begin{abstract}
Resumo
Neste artigo apresentamos resultados parciais de uma investigação a respeito da utilização de uma Trajetória Hipotética de Aprendizagem (THA) no planejamento de ações a serem implementadas em uma atividade de Modelagem Matemática. A pesquisa, de natureza qualitativa de cunho interpretativa, tomou como objeto de estudo as informações coletadas na implementação de uma THA com um grupo de professores de Matemática e estudantes de Licenciatura em Matemática, participantes de um minicurso. O objetivo específico é de analisar as produções apresentadas pelos participantes em uma atividade de modelagem, especificamente no que diz respeito a cada uma das fases da Modelagem: Inteiração, Matematização, Resolução, Interpretação dos Resultados e Validação, e relacioná-las à THA previamente planejada, observando como cada uma dessas fases foram antecipadas hipoteticamente. Como resultados parciais, inferimos que o planejamento, por meio de uma THA, pode configurar-se como instrumento norteador do trabalho docente, bem como uma estratégia de formação docente para o trabalho com Modelagem Matemática.
\end{abstract}

Palavras-chave: Educação Matemática. Trajetória Hipotética de Aprendizagem. Modelagem Matemática em Sala de Aula.

\begin{abstract}
In this paper, we present partial results of an investigation about the use of a Hypothetical Learning Trajectory (HLT) in the planning of actions to be implemented in the Mathematical Modeling activity in the classroom. The qualitative research had as study object the information collected in an implementation of the HLT performed with a group of Mathematics teachers and undergraduate students in Mathematics, participants of a short-term course. The specific objective of this work is to analyze the productions presented by the participants in an modeling activity specifically with respect to each of the phases of Modeling: Interaction, Mathematics, Resolution, Interpretation of Results and Validation, and to relate it to the previously elaborated HLT, observing

\footnotetext{
* Doutora em Ensino de Ciências e Educação Matemática pela Universidade Estadual de Londrina (UEL). Docente do Depto. de Matemática da Universidade Estadual de Londrina (UEL), Londrina-PR, Brasil. Endereço: Rodovia Celso Garcia Cid, PR 445, Km 380, Cx. Postal 10.011 - Campus Universitário, Depto de Matemática, CCE, UEL, Londrina-PR, Brasil, CEP: 86057-970. E-mail: pamelauel@ gmail.com.

** Doutora em Ensino de Ciências e Educação Matemática pela Universidade Estadual de Londrina (UEL). Docente do Departamento Acadêmico de Matemática e do Programa de Pós-Gradução em Ensino de Matemática da Universidade Tecnológica Federal do Paraná (UTFPR), Londrina, Paraná, Brasil. Endereço para correspondencia: Avenida dos Pioneiros, 3131, Londrina, Paraná, Brasil, CEP: 86036-370. E-mail: karinasilva@utfpr.edu.br.
} 
with each of these phases what was hypothetically anticipated. As partial results, we infer that planning through an HLT can be configured as: an instrument of the theacher's work; a teacher formation strategy for teaching with Mathematical Modeling.

Keywords: Mathematics Education. Hypothetical Learning Trajectory. Mathematical Modeling in the classroom.

\section{Introdução}

Nos últimos anos temos observado, na Educação Matemática, o crescente interesse e a quantidade de pesquisas relacionadas à formação de professores, que apresentam quase sempre uma intenção comum: superar as perspectivas tradicionais de ensino e de formação de docente. Além disso, observa-se também, nos documentos orientadores para o ensino, a perspectiva de um currículo contextualizado que considere as realidades da escola e do seu alunado, que seja voltado para formação de competências.

Muitas vezes, associado aos objetivos "conteudistas" dos currículos, o que é valorizado no ensino, quase sempre, é o cumprimento de uma lista praticamente "inatingível" de conteúdos matemáticos, ao passo que as questões relativas às competências ficam desvalidas. Ao sugerir uma aprendizagem por competências, os conteúdos matemáticos deixam de ser os "protagonistas" de uma meta de aprendizagem e passam a ser "o caminho" para a construção de um conhecimento contextualizado e flexível. Essa subversão não diminui a importância da aprendizagem de conteúdos em si, pelo contrário, ao constituir-se como caminho, faz com que ela seja valorizada e enriquecida de significados.

Na tentativa de sugerir uma possível "subversão", para uma educação matemática de qualidade, entra em cena, alguns elementos fundamentais para a reflexão: o uso de diferenciadas estratégias metodológicas; o delineamento dos objetivos para o ensino (relativos aos conteúdos, mas, também, às competências); o entendimento de uma avaliação que valorize o conhecimento; a utilização de tarefas contextualizadas; o conhecimento da realidade local, social e individual da escola e dos estudantes. Consequentemente, faz-se necessário refletir, nesse conjunto de elementos, a importância da formação docente e o modo de organização de práticas escolares.

Nessa perspectiva, consideramos fundamental a importância do planejamento e neste artigo apresentamos resultados parciais de uma pesquisa que teve como base a implementação de uma Trajetória Hipotética de Aprendizagem (THA) como um elemento norteador da prática docente, no planejamento de ações a serem desenvolvidas em sala de aula. Também foram considerados, para este estudo, o uso da Modelagem Matemática na Educação 
Matemática como estratégia de ação; a concepção de aprendizagem matemática dela subjacente; o desenvolvimento de competências associadas ao seu processo; e como consequência natural do processo, a aprendizagem matemática.

Trazemos para reflexão o uso da Modelagem Matemática como alternativa para superar práticas tradicionais de ensino e o uso da THA como recurso na preparação e antecipação hipotética do trabalho a ser realizado pelo docente ao trabalhar com THA. Esta pesquisa contou com a preparação de uma $\mathrm{THA}^{1}$ (pelas pesquisadoras) que serviu como recurso para implementar uma atividade de Modelagem Matemática. O objetivo específico deste artigo é de apresentar uma análise das produções apresentadas pelos participantes da pesquisa e relacioná-las à THA, que foi previamente elaborada pelas pesquisadoras, a qual deu origem às atividades de modelagem dos participantes.

\section{Trajetória hipotética de aprendizagem: um recurso para a formação docente}

A construção das práticas de salas de aula de um professor é orientada pelo conhecimento, teorias, concepções, experiências que possui e desenvolve ao longo de sua história de vida, mais especialmente, de sua formação. É no âmbito da formação docente que o professor desenvolve habilidades para estabelecer metas de ensino, realizar planejamentos, tomar decisões, reorganizar sua estratégia didática, seus procedimentos metodológicos.

Para promover a aprendizagem em sala de aula, as práticas docentes têm a função de guiar as ações dos estudantes, a interação no ensino e aprendizagem deve se dar em um ambiente de ir e vir, com situações que despertem significados para os alunos desenvolverem conhecimento de autoria própria, guiados pelo conhecimento historicamente produzido, com o auxílio do professor.

Martin Simon, um pesquisador americano introduziu, em 1995, a noção de Trajetória Hipotética de Aprendizagem (THA) para o ensino de Matemática. A intenção era propor uma reconstrução das práticas matemáticas construtivistas juntamente com a planificação do ensino, que consiste em um processo de planejar ações para um determinado período de ensino, constituído por metas, estratégias de ensino/aprendizagem, que organiza o quê e como deve ser ensinado.

Uma trajetória hipotética de aprendizagem - THA - é composta por três componentes:

\footnotetext{
${ }^{1}$ Os pressupostos teóricos da THA foram elementos norteadores do trabalho realizado pelas pesquisadoras. No entanto, os cursistas tiveram uma visão geral sobre o que é uma THA, apresentada teoricamente no minicurso, e a informação de que ela foi utilizada como base para a proposição da tarefa de Modelagem
} 
(1) o objetivo do professor sobre a aprendizagem de seus alunos; (2) o plano do professor para as tarefas $^{2}$ de aprendizagem; (3) as hipóteses do professor sobre um possível processo de aprendizagem: "uma suposição de como o pensamento e o entendimento dos alunos será colocado em ação no contexto de aprendizagem das atividades" (PIRES, 2009, p. 157). Simon e Tzur (2004, p. 93, tradução nossa) apresentam características de uma THA:

1. A construção de uma THA é baseada na compreensão do conhecimento atual dos estudantes envolvidos.

2. Uma THA é um veículo para o planejamento da aprendizagem de conceitos matemáticos específicos.

3. Tarefas matemáticas proporcionam ferramentas para promover a aprendizagem de determinados conceitos matemáticos e, assim, são uma parte fundamental do processo de ensino.

4. Devido à natureza hipotética e inerentemente incerta deste processo, o professor está frequentemente envolvido na modificação de todos os aspectos da THA.

Gómez, González e Lupiáñez (2007) argumentam que o conhecimento do professor, sua experiência e a literatura disponível são fontes básicas para que ele possa elaborar uma trajetória hipotética de aprendizagem que apoie seu planejamento. Segundo Steffe (2004)

\begin{abstract}
a construção de trajetórias hipotéticas de aprendizagem dos estudantes é um dos problemas mais desafiadores, porém urgentes, que a educação matemática enfrenta atualmente. É também um dos problemas mais empolgantes porque é a partir dela que podemos construir uma compreensão da matemática dos estudantes e como nós professores podemos utilizar de forma proveitosa essa matemática (STEFFE, 2004, p. 130 , tradução nossa).
\end{abstract}

Acreditamos que o planejamento é, em suma, uma das principais ações docente, pois subsidia as ações, tarefas, metas, objetivos a serem alcançados. A elaboração de uma THA considera a complexidade dos processos de ensino e aprendizagem e é potencial para desenvolver no professor a autonomia, segurança, o despertar para novas situações. Ao "levantar hipóteses" sobre os processos de ensino e de aprendizagem, o professor lança mão dos conhecimentos teóricos e práticos que possui, das estratégias metodológicas disponíveis para cumprimento de seus objetivos, das experiências vividas, dos conhecimentos que possui a respeito de seus alunos, constituindo uma rede de informações que lhe confere uma "antecipação" da aula que pretende propor.

Nesse sentido, entendemos que a elaboração de uma THA é uma estratégia que compete ao trabalho docente e faz com que o professor instrumente na forma de "planejamento" seu modo de lidar com uma determinada situação de ensino e aprendizagem.

\footnotetext{
2 Simon (1995) fala sobre "atividades de aprendizagem", no entanto chamaremos de "tarefas de aprendizagem" por entender que atividade diz respeito aos processos nos quais os alunos se envolvem a partir da uma proposta didática enunciada em uma tarefa.
} 


\title{
3 Modelagem Matemática em sala de aula: alguns encaminhamentos
}

Diversas pesquisas têm apontado o potencial para implementar Modelagem Matemática nas aulas de Matemática, seja nas aulas regulares (ALMEIDA; SILVA, 2010, BELTRÃO; IGLIORI, 2010, MELO; CHRISPINO, 2013, SILVA; BARONE; BASSO, 2016; SILVA, 2017; ALMEIDA; SILVA, 2017), seja em momentos extraclasse (ARAÚJO; CAMPOS, 2015; SCHROETTER et al., 2016; GEIGER; ÄRLEBÄCK; FREJD, 2016).

Nessas pesquisas, existe uma pluralidade de entendimento sobre Modelagem Matemática e os motivos de fazer uso dela em aulas de Matemática. De forma geral, os autores se fundamentam no fato de a Modelagem ser subsidiada por uma situação-problema que, a priori, não é da Matemática e da qual emerge um problema em que se busca uma solução matemática. Para Almeida e Silva (2017, p. 209), a “introdução e o uso da modelagem matemática nos diversos níveis de escolaridade e em diferentes cursos e disciplinas remete, entretanto, ao uso, à aplicação e à construção de conhecimento em Matemática”. Geiger, Ärlebäck e Frejd (2016, p. 208), todavia, argumentam que existem dois temas principais para a inclusão da Modelagem como parte da prática de sala de aula:

\begin{abstract}
O primeiro baseia-se na premissa de que a capacidade de modelar e encontrar soluções para situações relacionadas à vida é uma competência que pode servir ao indivíduo na vida cotidiana e no local de trabalho. O segundo apresenta a modelagem como um meio pelo qual os indivíduos constroem novos conhecimentos matemáticos ou reconstroem o conhecimento que já adquiriram ao se envolver com o processo de modelagem.
\end{abstract}

No nosso entendimento, quando nos referimos à Modelagem Matemática, consideramos atividades que têm como ponto de partida uma situação inicial (problemática) e como ponto de chegada uma situação final (solução para a situação inicial). Segundo Almeida e Ferruzzi (2009), o encaminhamento da situação inicial para a situação final requer do aluno a formulação de um problema e a definição de metas para sua resolução, a definição de hipóteses, a formulação de previsões e a apresentação de explicações e soluções para a situação em estudo, bem como a comunicação destas soluções e/ou explicações para outros.

Os conceitos e procedimentos matemáticos utilizados na busca pela solução para o problema se fazem presentes por meio de linguagem matemática, por meio de representação matemática que pode ser expressa por símbolos, diagramas, gráficos, expressões algébricas ou geométricas. Referimo-nos à representação matemática que emerge no desenvolvimento da atividade de Modelagem como modelo matemático.

$\mathrm{Na}$ literatura, existem pesquisadores que defendem o encaminhamento de uma atividade de Modelagem de forma cíclica, configurando-se o que se convencionou chamar de 
fases da Modelagem Matemática (BORROMEO FERRI, 2006; ALMEIDA; SILVA; VERTUAN, 2012; STILLMAN; BROWN; GEIGER, 2015). Almeida, Silva e Vertuan (2012) caracterizam fases relacionadas aos procedimentos necessários para configuração, estruturação e resolução de uma situação-problema. São elas: inteiração, matematização, resolução, interpretação dos resultados e validação.

A inteiração representa o primeiro contato do aluno com uma situação-problema que se pretende estudar. A matematização é a fase de transição de linguagens, de visualização e do uso de símbolos para realizar descrições matemáticas. A resolução consiste na obtenção do modelo matemático que descreve a situação. A interpretação dos resultados e validação são fases finais que visam, além da capacidade de aplicar o modelo matemático, o desenvolvimento da capacidade de avaliar o processo de construção do modelo e os diferentes contextos de suas aplicações. Essas fases podem apresentar constantes movimentos de idas e vindas, pois, às vezes, se tornam necessárias reformular ou analisar as fases anteriores.

Ainda que pesquisas relatem o potencial que atividades de Modelagem Matemática apresentam para a prática pedagógica, em sala de aula essa ação ainda é tímida. Dentre os aspectos que inibem a prática de Modelagem Matemática em sala de aula está o que Caldeira (2015) chama de "cumprir o currículo".

Com o propósito de superar essa inibição, Carlson et al. (2016, p. 122) assinalam que implementar Modelagem Matemática em sala de aula "envolve preparação, incluindo desenvolvimento da tarefa e antecipação das estratégias dos estudantes”. Stillman, Brown e Geiger (2015, p. 95) afirmam que antecipar estratégias possibilita prever o que "será útil matematicamente subsequentemente nas transições entre as fases do processo de modelagem”. Com isso, podemos conjecturar que a antecipação orienta o trabalho do professor e propomos que este planeje aulas com Modelagem seguindo uma trajetória hipotética. Para tanto, o professor pode levar em consideração uma aula conduzida segundo as fases supracitadas, na qual sua ação é a de orientar.

\section{Contexto da pesquisa e procedimentos metodológicos}

Para discutir uma possível articulação entre a construção de trajetórias hipotéticas de ensino e aprendizagem com Modelagem Matemática na formação inicial ou continuada propomos o desenvolvimento de uma tarefa de Modelagem durante um minicurso intitulado “É possível hipotetizar uma aula com modelagem matemática?" que ocorreu em 2016, durante um evento nacional (XII ENEM - Encontro Nacional de Educação Matemática), 
ministrado pelas autoras deste artigo que, doravante, identificamos por Prof_1 e Prof_2.

Como não conhecíamos de antemão os participantes, elaboramos previamente uma trajetória hipotética de uma atividade de Modelagem $^{3}$ que contemplou conhecimentos matemáticos abordados na Educação Básica, dado que o público alvo compreendia estudantes de Licenciatura em Matemática, professores da Educação Básica e professores de Graduação e Pós-Graduação.

Dos trinta e três participantes inscritos no minicurso:

- vinte e um eram formados em Matemática e doze estudantes de graduação;

- vinte e oito afirmaram que já haviam tido algum contato com Modelagem Matemática no curso de graduação ou em cursos de formação continuada;

- vinte afirmaram já terem desenvolvido atividade(s) de Modelagem Matemática com alunos.

Embora alguns tenham relatado insegurança nas primeiras atividades de modelagem por eles desenvolvidas, todos alegaram maior envolvimento dos alunos quando comparado com outras atividades.

Nesse contexto foi proposta uma tarefa ${ }^{4}$ de Modelagem (vide Quadro 1), cuja temática estava atrelada à velocidade de reação de pastilha de antiácido de acordo com a massa da mesma ${ }^{5}$. Para o desenvolvimento da proposta os participantes reuniram-se em nove grupos (os quais denominamos $\mathrm{G} 1, \mathrm{G} 2, \mathrm{G} 3, \ldots)$.

A coleta de informações para as análises que nos propusemos a realizar foi feita por meio de filmagem que nos possibilitou o áudio e a visualização dos gestos dos participantes, além dos registros escritos produzidos pelos grupos. Para nos referirmos aos participantes utilizamos a letra $\mathrm{A}$, um número que o diferencie dos colegas do grupo e o número do grupo. Por exemplo, A1G1 refere-se ao participante 1 do grupo 1; A2G2 refere-se ao participante 2 do grupo 2 e, assim sucessivamente.

Na seção seguinte "Da THA planejada" são relatados, de modo breve $e^{6}$, os principais elementos constituintes da THA que foi elaborada antes da execução do minicurso que serviu como contexto de campo. Na seção posterior, "Das análises realizadas" é então apresentada

\footnotetext{
${ }^{3}$ Para o desenvolvimento do minicurso, primeiramente traçamos uma trajetória hipotética de aprendizagem, tal como descrevemos na próxima seção "Da THA planejada".

4 Estamos chamando de tarefa a proposta inicial escrita. As ações realizadas pelos participantes no desenvolvimento, no lidar com a tarefa, é que são consideradas como atividades.

${ }^{5}$ Dados utilizados para a construção da referida tarefa foram coletados por alunos de um curso de Licenciatura em Química.

${ }^{6}$ A THA, tal como foi elaborada na íntegra, não poderá ser apresentada neste texto pelo fato de a quantidade limite de páginas não comportar todo o planejamento.
} 
uma análise das produções coletadas, à luz da análise de conteúdo (BARDIN, 2004), com a intenção de apresentar reflexões para o nosso objetivo específico. São elementos norteadores desta análise:

(1) as inferências sobre as fases da Modelagem Matemática desveladas por meio da análise dos registros escritos dos grupos;

(2) os modos pelos quais os participantes do minicurso abordaram uma situação proposta, destacando elementos observáveis nas fases de: Inteiração, Matematização, Resolução, Interpretação dos Resultados e Validação;

(3) uma articulação entre a THA elaborada e a dinâmica realizada, na tentativa de validar a ocorrência das hipóteses antecipadas na THA ou verificar aspectos não previstos que possam servir para "realimentar" a THA para uma nova aplicação;

(4) uma reflexão a partir da sistematização feita no minicurso, por meio dos diálogos que ocorreram.

Nesse artigo temos, como objetivo específico, apresentar uma análise a partir das produções apresentadas pelos participantes da atividade de Modelagem e relaciona-las à THA previamente elaborada. O objetivo geral da pesquisa, como um todo, é investigar a utilização de uma Trajetória Hipotética de Aprendizagem (THA) com uso da Modelagem Matemática na perspectiva da Educação Matemática.

\section{Da THA planejada}

Nesta seção apresentamos, de modo parcial, alguns elementos da THA que foi elaborada antes de sua implementação e que serviu como contexto de campo.

Os principais objetivos do planejamento do professor que pretende trabalhar com essa THA são: (a) articular uma situação proposta com a perspectiva da utilização da Modelagem Matemática; (b) ler e interpretar uma situação proposta; (c) compreender o processo de reação de uma pastilha de antiácido; (d) interpretar relações entre massa, tempo e velocidade; (e) descrever a situação proposta em termos matemáticos; (f) reconhecer e resolver problemas que podem ser solucionados por meio de função linear e outras relacionadas; (g) propor um problema de investigação, e apresentar solução para ele por meio do desenvolvimento de um modelo; (h) interpretar os resultados obtidos na resolução desse problema.

Visando o desenvolvimento de uma THA, sugere-se que sejam elaboradas algumas normas para a sua realização: um contrato pedagógico deve ser estabelecido entre professor e alunos, buscando planejar as ações para o bom andamento das aulas. 
No desenvolvimento dessa THA, é proposta uma tarefa aos participantes sobre a qual deverão lidar com ela, interpretando-a, inferindo sobre seu entendimento, estabelecendo relações entre a situação proposta e sua descrição matemática.

Nessa THA apresentamos uma proposta de tarefa de modelagem a ser desenvolvida em sala de aula por grupos de alunos que se utilizaram de "dados prontos" (DINIZ; BORBA, 2012). O intuito é inferir sobre os possíveis caminhos do desenvolvimento da atividade (tanto dos alunos como do professor) e possíveis objetos matemáticos que emergem em seu desenvolvimento por meio de uma THA. A proposta conta trabalhar com a seguinte tarefa (Quadro 1).

\section{Velocidade de reação de pastilha de antiácido}

Com o interesse de estudar Cinética Química, mais especificamente a velocidade de diluição de uma pastilha de antiácido com diferentes massas, um grupo de alunos realizou experimentos em um laboratório no qual utilizou pastilhas de antiácido com diferentes massas, $200 \mathrm{ml}$ de água e cronômetro.

Utilizando uma balança semi-analítica o grupo determinou a massa de cinco pastilhas e

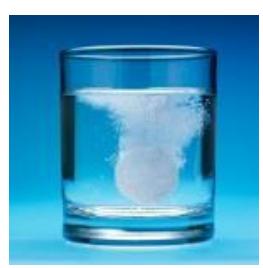
concluiu que uma pastilha de antiácido tem em média $4 \mathrm{~g}$. Em seguida, o grupo de alunos colocou a pastilha em um béquer com $200 \mathrm{ml}$ de água e cronometrou o tempo de diluição.

Considerando que $V=\frac{m}{t}$, determinaram a velocidade de reação da pastilha.

O grupo realizou os mesmos procedimentos para outras massas que foram obtidas com a "quebra" de pastilhas maiores e representou os dados coletados em uma tabela (Tabela 1).

Tabela 1 - Uma "possível" coleta de dados

\begin{tabular}{ccc}
\hline $\begin{array}{c}\text { Massa da pastilha } \\
(\mathbf{g})\end{array}$ & Tempo $(\mathbf{s})$ & $\begin{array}{c}\text { Velocidade da } \\
\text { reação }(\mathbf{g} / \mathbf{s})\end{array}$ \\
\hline 4 & 42 & 0,09 \\
1 & 32 & 0,03 \\
0,7 & 25 & 0,02 \\
0,5 & 23 & 0,016 \\
0,2 & 22 & 0,01 \\
\hline
\end{tabular}

Fonte: Dados coletados em laboratório (2016).

A partir dessa situação proponha um problema a ser investigado e resolva-o.

Quadro 1 - Tarefa de Modelagem

Fonte: Da pesquisa (2016).

Nos Quadros 2, 3 e 4 apresentamos considerações hipotéticas sobre como cada uma das fases da Modelagem Matemática pode se apresentar no desenvolvimento realizado pelos alunos.

\begin{tabular}{|l|l|l|}
\hline \multicolumn{1}{|c|}{ Hipótese 01: } & \multicolumn{1}{c|}{ Hipótese 02: } & \multicolumn{1}{c|}{ Hipótese 03: } \\
\hline • Inteiração & $\bullet$ Inteiração & $\bullet$ Inteiração \\
• Matematização & $\bullet$ Matematização & $\bullet$ Matematização \\
• Resolução & • Interpretação dos Resultados e Validação & • Interpretação dos \\
• Interpretação dos Resultados & • Matematização & Resultados e Validação \\
e Validação & • Resolução & • Resolução \\
& $\bullet$ Interpretação dos Resultados e Validação & \\
\hline
\end{tabular}

Quadro 2 - Hipóteses sobre os "movimentos" do processo de Modelagem

Fonte: Da pesquisa (2016) 


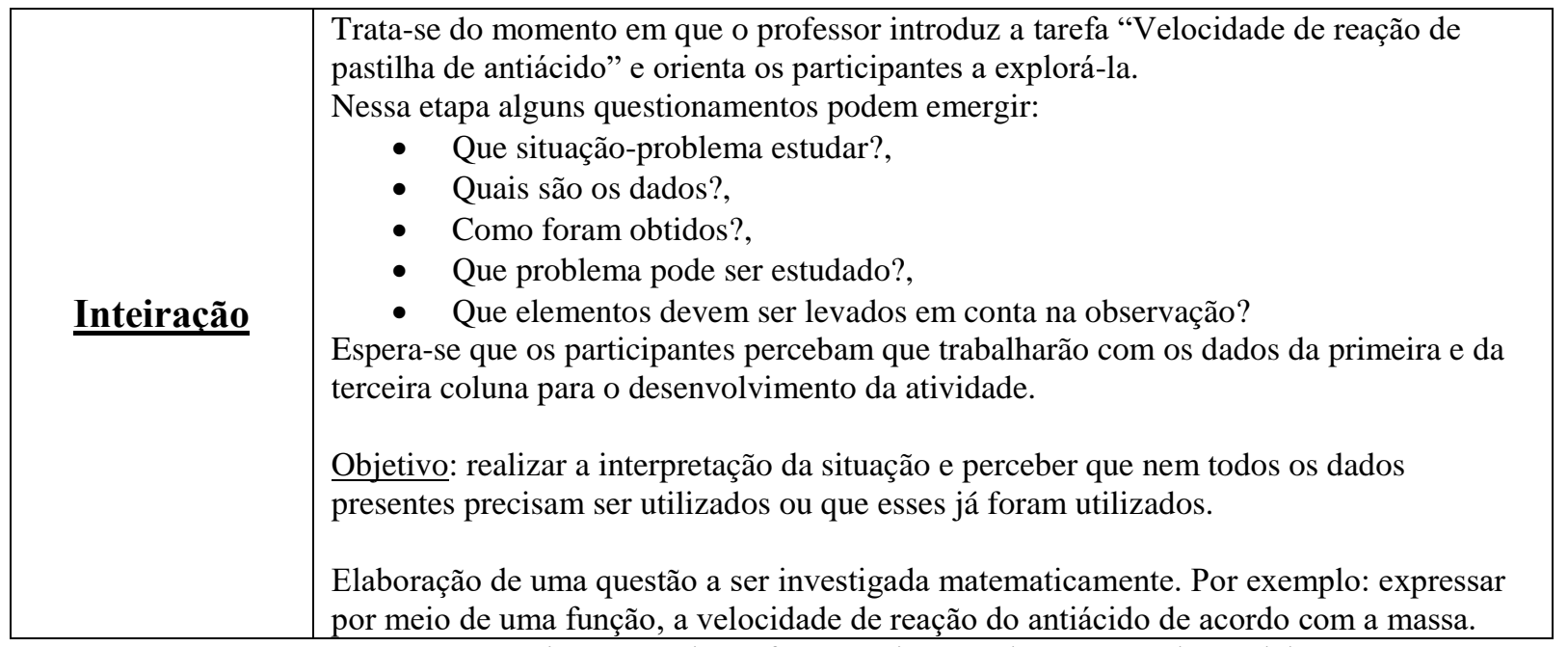

Quadro 3 - Hipóteses sobre a fase "Inteiração" do processo de Modelagem

Fonte: Da pesquisa (2016)

\begin{tabular}{|c|l|}
\hline \multirow{5}{*}{ Matematização } & $\begin{array}{l}\text { Nessa etapa, uma vez definido o problema, os participantes lançarão mão das estratégias } \\
\text { e conhecimentos matemáticos que possuem para responder: } \\
\bullet \quad \text { Quais são as hipóteses e variáveis da situação? } \\
\bullet \quad \text { Que regularidades matemáticas podem ser observadas a partir dos dados? } \\
\text { Uma possibilidade é a de representar os pontos (massa, velocidade) no plano cartesiano. } \\
\text { A partir da percepção do comportamento dos dados, considerar por hipótese que eles se } \\
\text { ajustam a uma reta: } V(m)=a m+b, \text { em que: } V \text { é a velocidade de reação }(\mathrm{g} / \mathrm{s}) \text { em } \\
\text { função da massa do antiácido } m(\mathrm{~g}) .\end{array}$ \\
\hline
\end{tabular}

Quadro 4 - Hipóteses sobre a fase "matematização" do processo de Modelagem Fonte: Da pesquisa (2016)

No Quadro 5 apresentamos nossas considerações hipotéticas sobre "Resolução e Interpretação dos Resultados e Validação" que o processo de Modelagem Matemática pode se apresentar no desenvolvimento realizado pelos alunos.

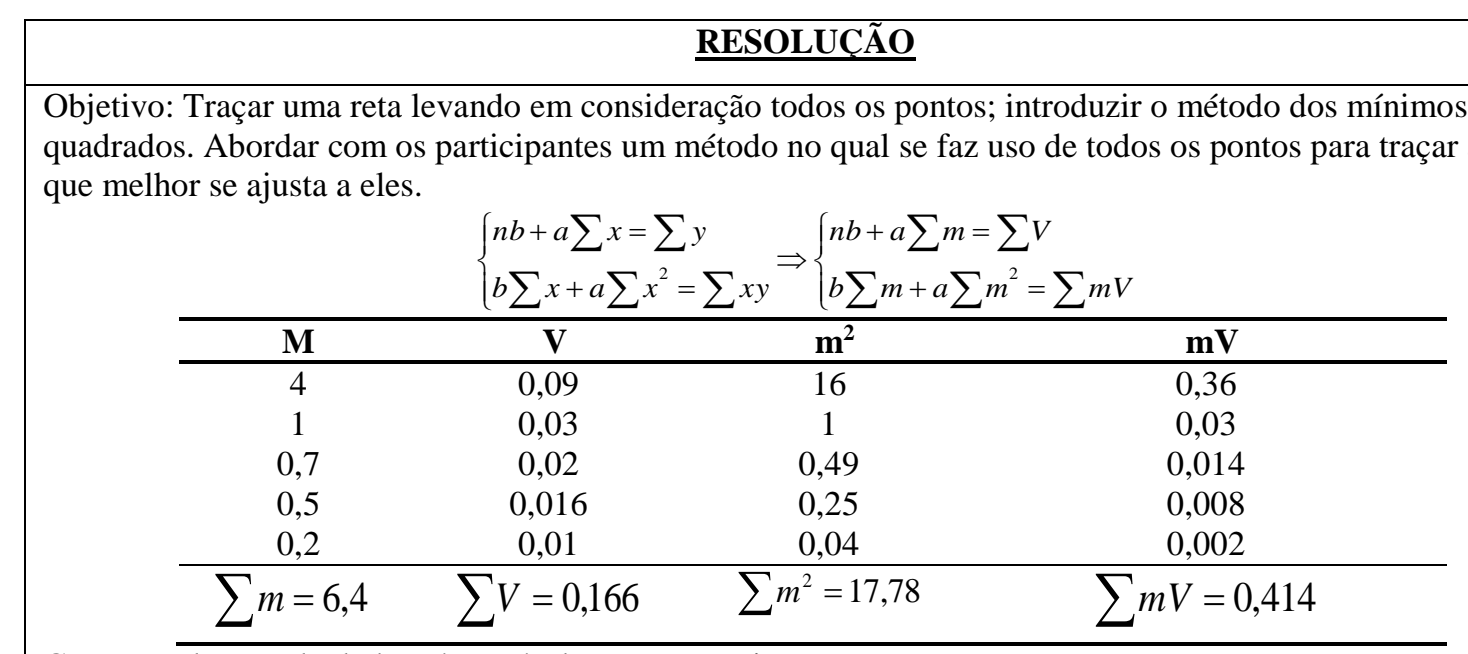

Com os valores calculados, é possível montar um sistema:

$$
\left\{\begin{array} { l } 
{ 5 b + 6 , 4 a = 0 , 1 6 6 } \\
{ 6 , 4 b + 1 7 , 7 8 a = 0 , 4 1 4 }
\end{array} \Rightarrow \left\{\begin{array}{l}
32 b+40,92 a=1,0624 \\
-32 b-88,9 a=-2,07
\end{array}\right.\right.
$$

Assim $a \cong 0,021$ e $b \cong 0,00632$, obtendo o modelo matemático $V(m)=0,021 m+0,00632$ 


\section{INTERPRETAČ̃̃O DOS RESULTADOS E VALIDAÇ̃̃O}

- O modelo matemático obtido corresponde à situação em estudo?

- Qual é a solução do problema?

- Realizar a validação do modelo matemático a partir da expressão algébrica, inserindo uma coluna na tabela e realizando os cálculos:

Tabela 4 - Validação do modelo matemático

\begin{tabular}{ccc}
$\begin{array}{c}\text { Massa da pastilha } \\
(\mathbf{g})\end{array}$ & $\begin{array}{c}\text { Velocidade da } \\
\text { reação }(\mathbf{g} / \mathbf{s})\end{array}$ & $\begin{array}{c}\text { Velocidade da reação pelo } \\
\text { modelo matemático }(\mathbf{g} / \mathbf{s}) \\
V(m)=0,021 m+0,00632\end{array}$ \\
\hline 4 & 0,09 & 0,09032 \\
1 & 0,03 & 0,02732 \\
0,7 & 0,02 & 0,020102 \\
0,5 & 0,016 & 0,01682 \\
0,2 & 0,01 & 0,01052 \\
\hline
\end{tabular}

Fonte: Dados coletados em laboratório

Quadro 5 - Abordagem 3 sobre "Resolução e Interpretação dos Resultados e Validação" do processo de Modelagem

Fonte: Da pesquisa (2016)

\section{Das análises realizadas}

Com o objetivo de apresentar reflexões sobre as produções dos participantes em uma atividade de Modelagem Matemática e relacioná-las à THA planejada, , escolhemos convenientemente $^{8}$ dois grupos para serem analisados: Grupo 3 (G3) e Grupo 6 (G6). Nos quadros 6 e 7 são apresentados extratos das produções de G3 e G6, respectivamente, para tecermos nossas considerações.

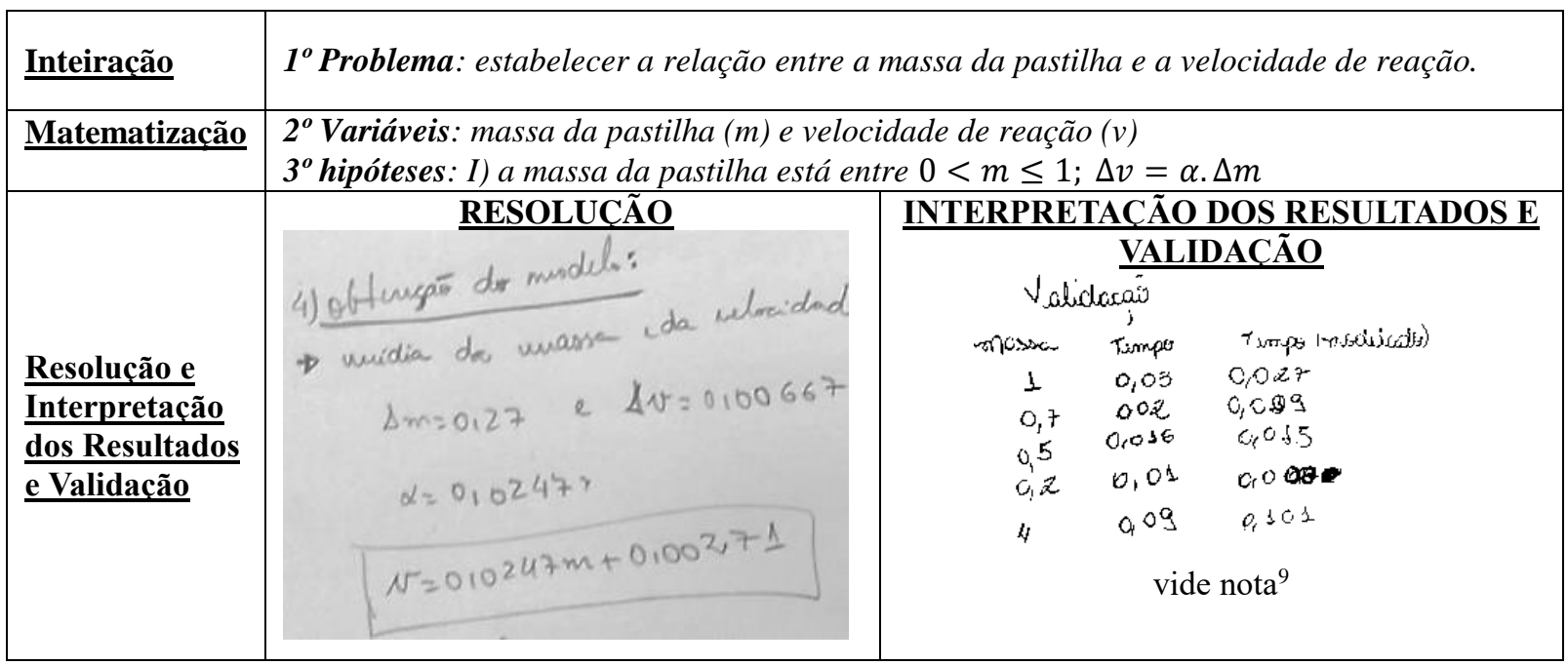

\section{Quadro 6 - Produções de G3}

Fonte: Da pesquisa (2016) - Registro escrito de G3

\footnotetext{
${ }^{7}$ Para ilustrar os tipos de abordagens que foram considerados na THA planejada pelas autoras antes da implementação, apresentamos apenas um exemplo: a abordagem de número 3.

${ }^{8}$ Essa escolha se deu, porque os grupos analisados apresentam informações que nos dão condições de promover a reflexão desejada.

"A coluna descrita como "tempo" diz respeito à "velocidade". Acreditamos que tenha sido uma distração do descritor do grupo.
} 
No que diz respeito às hipóteses sobre os "movimentos" da Modelagem, descritos no Quadro 2 da THA, identificamos que G3 seguiu exatamente o movimento conforme a hipótese 1, qual seja: Inteiração; Matematização; Resolução; Interpretação dos Resultados e Validação (vide Quadro 6).

De posse da tarefa proposta, a inteiração de G3 ficou por conta do que considerariam para investigar a situação problemática "velocidade de reação da pastilha de antiácido", ou seja, diante da tabela 1 com dados sobre a massa da pastilha, o tempo de diluição e a velocidade de reação, identificar aqueles que seriam considerados para desenvolver a atividade, conforme transcrição das considerações compartilhadas:

A1G3: Diferente deles [referindo-se à abordagem realizada pelo G2], a gente considerou a massa da pastilha e a velocidade de reação. E o nosso problema é o seguinte: dependendo da massa da pastilha e velocidade de reação dela.

Prof_1: Então é uma relação entre massa e velocidade?

AlG3: Isso.

(Diálogo entre professor e aluno, 2016).

O que fica evidente é que a primeira ação dos integrantes do grupo foi identificar que dados considerariam e, de posse desses dados, definiram um problema a ser estudado. Segundo Almeida e Ferruzzi (2009), a formulação do problema e a definição de metas para sua resolução são os primeiros encaminhamentos para partir da situação inicial rumo a uma situação final.

No que diz respeito às hipóteses sobre a fase "Inteiração" da Modelagem descritas no Quadro 3 da THA, identificamos que: a pergunta "Que situação-problema estudar?" fez sentido para o grupo uma vez que, G3 cumpre o objetivo de realizar a interpretação da situação e percebe que "nem todos os dados presentes precisam ser utilizados". Consequentemente, elabora uma questão a ser investigada matematicamente, qual seja a de: "estabelecer a relação entre a massa da pastilha e velocidade de reação".

Outros grupos analisados nessa fase apresentaram outras questões como: "Determinar a massa em função do tempo"; "Determinar o tempo em função da massa"; "Determinar a velocidade de reação em função do peso humano"; "Determinar a velocidade de reação de duas pastilhas". No entanto, a hipótese "mais forte" que tivemos ao elaborar a THA foi de que os grupos estabeleceriam o seguinte problema: "Determinar a velocidade em função da massa", traduzido ao nosso olhar pelo desenvolvimento apresentado por G3.

Com o problema definido, a matematização foi iniciada com o grupo considerando algumas informações quantitativas apresentadas na Tabela 1 com a intenção de visualizar nos dados alguma regularidade, conforme transcrição: 
A1G3: Então a gente pegou esses dados e foi fazer um gráfico [Figura 1] para ver o que dá. Então a gente viu que para alguns valores não dava para ser realizado, quando chegava em quatro ela dava um salto muito alto. Dai não dava para se trabalhar. E o que a gente fez? A gente elaborou uma hipótese que a pastilha está entre zero, é maior do que zero e menor do que um. A gente trabalhou nesse intervalo, e que essa reação nesse intervalo seria da seguinte forma: delta vé igual a alfa, coeficiente angular, vezes a variação da massa. E a partir desse momento nós conseguimos modelar (Diálogo entre professor e aluno, 2016).

Embora tenham representado os dados no plano cartesiano e traçado uma curva de tendência para os mesmos, conforme Figura 1, os integrantes de G3 consideraram que, para a abordagem do problema, somente o intervalo $(0,1]$ deveria ser utilizado, pois "quando chegava em quatro ela dava um salto muito alto" (Gravação do diálogo entre professor e aluno, 2016).

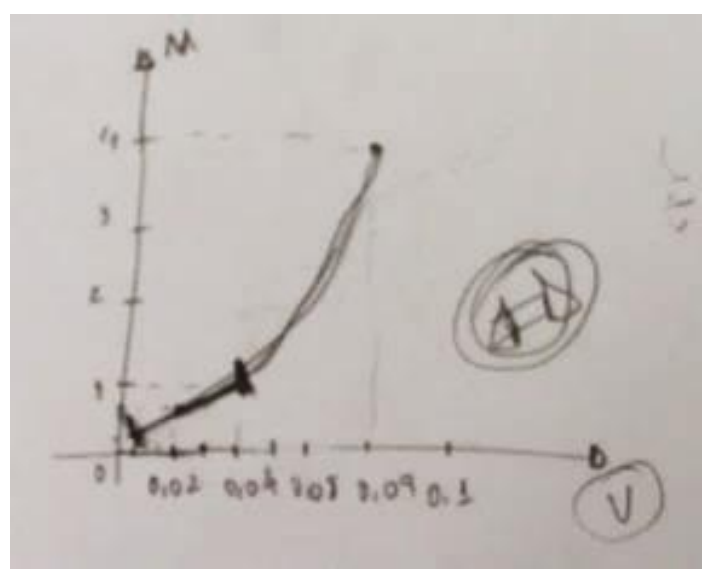

Figura 1 - Representação gráfica para os dados Fonte: Registro de G3 (2016).

A visualização dos dados no plano cartesiano fez com que os integrantes de G3 sentissem necessidade de realizarem simplificações para a dedução de um modelo matemático, limitando o intervalo de massa para a pastilha de antiácido. As simplificações, segundo Borromeo Ferri (2006), auxiliam no estudo do problema em atividades de Modelagem Matemática.

No que diz respeito às hipóteses sobre a fase "Matematização" em uma atividade de Modelagem descritas no Quadro 4 da THA, identificamos que G3 ao explorar as hipóteses e variáveis da situação: lança mão de estratégias conhecidas como "a elaboração de um gráfico" para compreender o comportamento dos dados da Tabela 1 e, também, "realiza a seleção de parte dos dados" para trabalharem com um modelo conhecido por eles, o da função afim, carregado dos procedimentos tais como "determinação do coeficiente angular da reta".

Considerando as hipóteses de que a massa pertence ao intervalo $(0,1]$ e de que "reação nesse intervalo seria da seguinte forma: delta v é igual a alfa, coeficiente angular, vezes a variação da massa" (Diálogo entre professor e aluno, 2016), uma função polinomial 
de primeiro grau obtida pela relação $\Delta v=\alpha . \Delta m$ foi considerada e modelada pelo grupo. $\mathrm{O}$ modelo matemático obtido na fase de resolução foi expresso por $v=0,0247 m+0,00271$, em que $m \in(0,1]$ representa a massa da pastilha (em gramas) e $v \in(0,0,02741]$ representa a velocidade de reação da pastilha (em gramas/segundos). Para obter os parâmetros da função polinomial, os alunos realizaram procedimentos matemáticos com os dados apresentados na Tabela 1, conforme explicado no compartilhamento de resultados e apresentados na transcrição:

A2G3: Entre zero e um a pastilha se comporta de forma linear, né? A variação da velocidade vai ser uma constante vezes a variação da massa. A partir dai a gente fez uma média das massas, da variação das massas e da velocidade, fez uma média de todas elas, considerada cada velocidade e cada massa, ai fez uma média delas e usando essa média, a gente chegou num modelo, né? Que dai a gente substituiu lá e achou o alfa e tudo. [...]

Prof_2: Vocês obtiveram uma expressão algébrica?

A1G3, A2G3: Sim!

A3G3: Pois é.

Prof_2: Ecomoficou?

A3G3: Mas sem considerar massa de quatro gramas. Só entre zero e um. Nós discutimos nossa massa só entre zero e um.

Prof_2: Só entre zero e um?

A3G3: Essa foi a hipótese.

Prof_2: E como ficou o modelo? Vocês querem escrever aqui? Alguém pode escrever?

AlG3: Pode escrever, professora. A velocidade em relação a massa, $v$ de $m$ igual a zero virgula zero dois quatro sete $m$ mais zero virgula zero outro zero vinte e sete um.

(Gravação do diálogo entre professor e aluno, 2016).

Enquanto A1G3 e A3G3 apresentam a expressão algébrica do modelo matemático para o estudo da velocidade de reação da pastilha em função da massa, A2G3 continua a realizar cálculos com o intuito de realizar a validação, conforme transcrição:

A2G3: Com esse modelo a gente chegou bem próximo. [...]

A1G1: E o mé maior que zero e menor que um, menor ou igual a um.

A2G3: Eu testei para o quatro agora, deu zero vírgula um zero um, bem próximo né?

Prof_2: E o experimental deu zero vírgula zero nove.

A3G3: Isso.

Prof_2: Então depende se considerar o comportamento é linear do domínio de zero a quatro... A3G3: É válido.

(Gravação do diálogo entre professor e aluno, 2016).

No que diz respeito às hipóteses sobre a fase "Resolução e Interpretação dos Resultados e Validação" da Modelagem descritas no Quadro 5 da THA, identificamos que G3 passa por um processo mais próximo da Abordagem 2, que foi considerada como hipotética na THA (que diz respeito à construção de um modelo linear).

Ao explorar regularidades e alguma representação matemática para a situação, G3 cumpre o objetivo de saber que: (i) a taxa de variação da velocidade em relação à massa determina o coeficiente angular da reta; (ii) são suficientes dois pontos para determinar essa 
taxa de variação; (iii) a partir desses procedimentos seria possível fornecer um modelo de função afim para representar a situação em estudo.

Com a validação, os integrantes de G3 realizam uma interpretação matemática para a situação por meio do estudo da velocidade de reação da pastilha de antiácido em relação à massa. Esse momento de validação é importante para que o desenvolvimento da Modelagem pelo grupo se torne completo.

Apesar de G3 não ser um grupo muito experiente com tarefas de Modelagem, apresenta um registro por meio de uma possível tabela (vide Quadro 6) que configura sua validação do problema estudado. Na THA planejada é prevista a necessidade de que os grupos passassem por este processo. No entanto, o seu valor não está explicitamente em prever que modelos os alunos podem apresentar, mas sim em orientar o docente com questões norteadoras que ajudem no processo tal como: "O modelo matemático obtido corresponde à situação em estudo? Qual é a solução do problema?”.

Os integrantes de G6, todavia, levaram em consideração os dados relativos à massa da pastilha de antiácido e o tempo de reação. Com isso, definiram o problema: Qual o tempo de diluição de uma pastilha de dois gramas, considerando o mesmo volume de água do experimento? O encaminhamento seguido por G6 é apresentado no Quadro 7.

\begin{tabular}{|c|c|}
\hline$\underline{\text { Inteiracão }}$ & $\begin{array}{l}\text { Qual o tempo de diluição de uma pastilha de dois gramas, considerando o mesmo volume } \\
\text { de água do experimento? }\end{array}$ \\
\hline Matematização & $\begin{array}{ll}\checkmark & \text { Simplificação } \\
\checkmark & \text { Variáveis: } \\
& \text { Independente (massa da pastilha em g) } \\
& \text { Dependente: } t \text { (tempo em segundos) } \\
\checkmark & \text { Hipótese: a relação matemática que expressa o tempo de diluição da pastilha } \\
& \text { em função de sua massa pode ser definida apenas no intervalo de } 0 \text { a } 4 \mathrm{~s}\end{array}$ \\
\hline$\frac{\text { Resolucão e }}{\text { Interpretação dos }}$ & 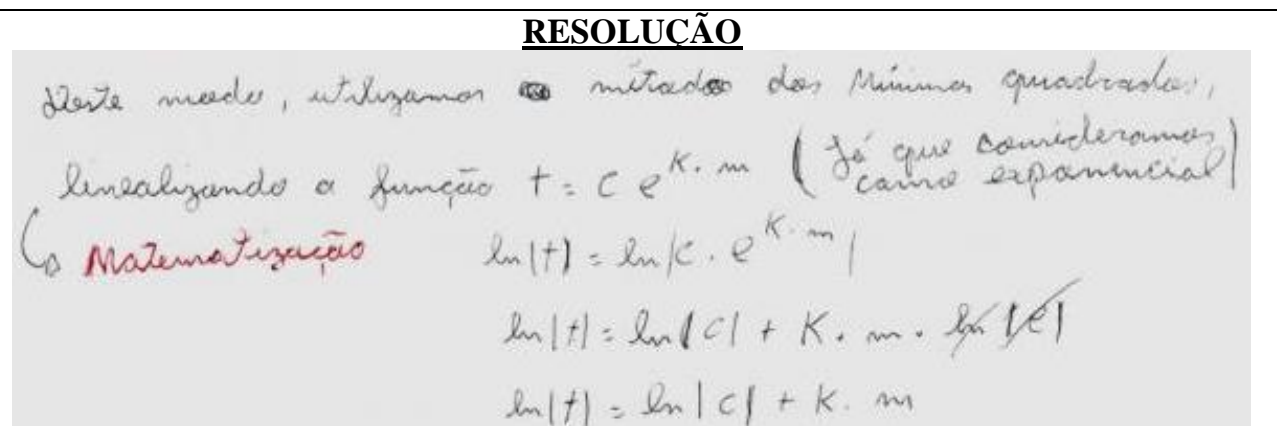 \\
\hline$\frac{\text { Resultados e }}{\text { Validação }}$ & $\begin{array}{ll} \begin{cases}A \varepsilon n^{2}+B \varepsilon n=\sum t m & A=K \\
A \varepsilon n+B A=\varepsilon F & B=\ln |C| \\
& F=\ln |\pi|\end{cases} \\
\left(\frac{16,648-B 5}{6,4}\right) \cdot 1+, 78+6,4 B=22,855\end{array}$ \\
\hline
\end{tabular}




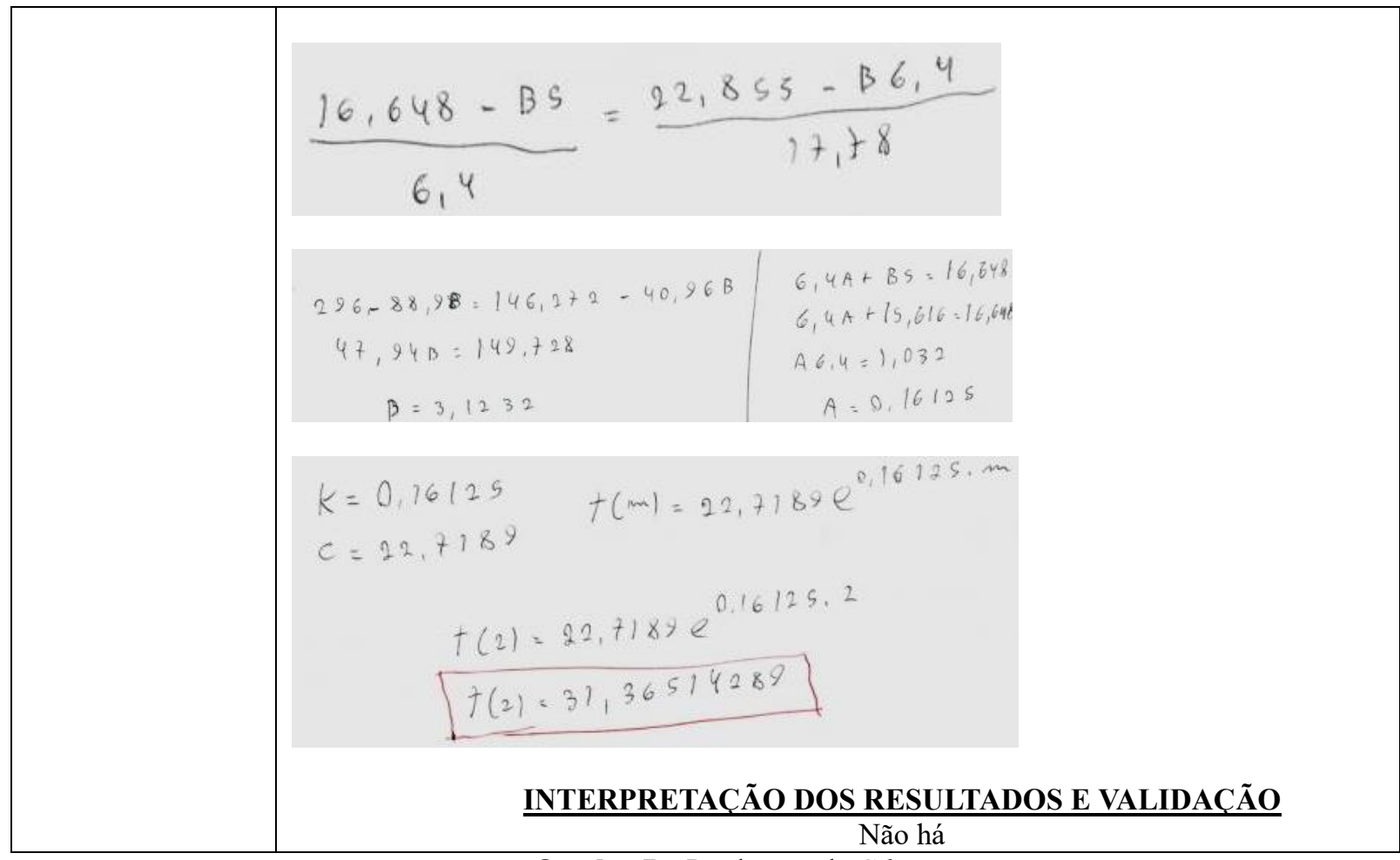

Quadro 7 - Produções de G6

Fonte: Da pesquisa - Registro escrito de G6 (2016).

Sobre o "movimento" do desenvolvimento da Modelagem, G6 não seguiu nenhuma das hipóteses levantadas na THA. A partir da análise que realizamos podemos inferir que cumpriram as fases Inteiração, Matematização e Resolução, porém, não realizaram a fase Interpretação dos Resultados e Validação (vide Quadro 7).

Para definir o que de fato seria um problema a ser resolvido via tarefa de Modelagem Matemática proposta, o grupo levou em consideração alguns apontamentos sobre a situação e a sobre a Matemática que poderiam subsidiá-la conforme transcrição compartilhada pelo grupo sobre o encaminhamento:

A1G6: Bom, no primeiro momento a nossa intenção foi olhar os dados e ver que eles não estão igualmente espaçados e ai não dava para ver se era exponencial ou linear diretamente dos dados. Ai a gente foi investigar a situação. E aí averiguamos essas questões, a superficie das pastilhas. Se a gente considerasse duas pastilhas de quatro, qual seria o tempo? Então com todas as questões que a gente foi discutindo, acho que em quase todos os outros grupos, a gente, é... formulou o seguinte problema: Qual é o tempo de diluição de uma pastilha de dois gramas, considerando o mesmo volume de água e o mesmo recipiente do experimento?.

(Gravação do diálogo entre professor e aluno, 2016).

Os integrantes de G6 já tinham alguma experiência com o desenvolvimento de atividades de Modelagem Matemática, desse modo, a primeira ação foi a busca por uma interpretação matemática para os dados, mesmo antes da definição do problema. Como de imediato não identificaram uma Matemática que pudesse representar a situação - não dava para ver se era exponencial ou linear diretamente dos dados (Gravação do diálogo entre 
professor e aluno, 2016) - os integrantes se inteiraram da mesma na busca de compreensão do fenômeno: o tempo de reação da pastilha em relação à superfície de contato. Todavia, para responder à questão elucidada pelo grupo, algumas hipóteses foram consideradas, conforme transcrição:

A1G6: Daí a gente iria considerar o mesmo recipiente e o mesmo volume. E aí trabalhando com... a hipótese de que é uma função exponencial definida no intervalo de zero a quatro gramas, então assim consideramos o domínio de zero a quatro gramas, né?

(Gravação do diálogo entre professor e aluno, 2016).

Conforme previsto na THA (Quadro 3), na fase "Inteiração" da Modelagem, G6 explora a tarefa e questiona-se sobre qual situação-problema estudar, seleciona parte dos dados, determina um problema e percebe que não necessita utilizar de todos os dados da Tabela 1. Diferente do previsto, G6 opta por trabalhar com a primeira e segunda colunas da Tabela 1, mas não deixa de elaborar uma questão a ser investigada, qual seja a de "Determinar o tempo de reação em função da massa de 2 gramas”.

No que diz respeito às hipóteses sobre a fase "Matematização" (Quadro 4) identificamos que, ao explorar “Quais são as hipóteses e variáveis da situação?”, G6, por já ter tido experiências com Modelagem, sinaliza a necessidade de realizar simplificação dos dados, estabelecer as hipóteses e variáveis do problema a serem estudadas. No que concerne “Que regularidades matemáticas podem ser observadas a partir dos dados?”, o grupo G6 realiza a seleção de parte dos dados para trabalharem com um modelo conhecido por eles.

A matematização é realizada por meio da hipótese considerada por G6 de que um modelo exponencial poderia representar o comportamento dos dados no intervalo em que $m \in$ $(0,4)$. Para a resolução e dedução de um modelo matemático, G6 utilizou o método dos mínimos quadrados, conforme apresentado no Quadro 7 e na transcrição:

A1G6: E utilizando o método dos mínimos quadrados a gente encontrou a função t de migual a vinte e dois vírgula é... vinte e dois vírgula setenta e dois zero quatro vezes Euler elevado a zero dezesseis onze noventa e sete vezes $m$. E ai para o tempo, para a massa de dois gramas deu trinta e um vírgula trinta e seis... e aí, aproximadamente, trinta e um vírgula trinta e seis segundos [...]

(Gravação do diálogo entre professor e aluno, 2016).

Com o modelo matemático deduzido, a ação dos alunos foi obter uma solução para o problema. A solução para o problema é entendida, segundo Almeida, Silva e Vertuan (2012, p. 18), como uma interpretação em que "o aluno se depara com a necessidade de comparação e distinção de ideias, generalização de fatos, articulação de conhecimentos de diferentes áreas". No entanto, os integrantes se depararam com um impasse, pois, ao compararem a solução com os dados apresentados na Tabela 1, o tempo de diluição de uma pastilha de dois gramas era 
inferior ao de um grama, conforme transcrição:

A1G6: [...] e ao validar esse modelo, a gente utilizou o Excel para ajustar alguns pontos, colocar a linha de tendência e aí a gente viu que a exponencial tinha um ponto que estava bem discrepante que era um e trinta e dois. Ai ao olhar o r quadrado dele comparando com o valor de outros a linear e a logarítmica, a gente viu que a função exponencial era a mais adequada acho que da... de uma linear de uma logarítmica. E aí a gente validou e eu acho que o ponto que ficou meio for mesmo foi quando a massa é igual a um grama que modelado ficou vinte e seis vírgula sessenta e nove segundos.

(Gravação do diálogo entre professor e aluno, 2016).

Sobre a fase "Resolução e Interpretação dos Resultados e Validação" da Modelagem descrita no Quadro 5 da THA, identificamos que G6 passa por um processo que não se aproxima das abordagens previstas, a não ser pelo fato de utilizar o método dos mínimos quadrados. No momento da discussão dos grupos, G6 sinaliza que faltou a "Interpretação dos Resultados e Validação". Na oportunidade de ter mais tempo do que o disponível para a atividade de Modelagem, a atividade de G6 poderia ter sido orientada segundo previsto na THA e, com a devida instrução do docente, a questionar-se "O modelo matemático obtido corresponde à situação em estudo? Qual é a solução do problema?”.

Com este caso observado de G6, obtém-se a oportunidade de refinar a trajetória planejada no sentido de prever outro movimento, tal como uma hipótese 4: "Inteiração; Matematização; Resolução Interpretação dos Resultados; Matematização; Resolução Interpretação dos Resultados e Validação".

\section{Considerações preliminares}

Embora, geralmente uma THA considere mais de uma tarefa de aprendizagem e a THA que aqui apresentamos utiliza-se de apenas uma tarefa, entendemos as variadas fases nas quais os participantes se envolvem ao realizar uma atividade de Modelagem Matemática. Muitas outras hipóteses podem ser previstas em uma THA, que diga respeito aos muitos processos pelos quais os alunos podem passar ao se envolverem em uma atividade de Modelagem. O importante desse instrumento não é apenas fazer previsões de tudo que pode ocorrer, mas sim dar liberdade para que possam ocorrer. Além disso, fatores relevantes para a condução e orientação da atividade estão presentes na trajetória apresentada, quais sejam as perguntas motivadoras e orientadoras do processo.

Pode existir, por exemplo, uma quantidade de combinações possíveis entre o problema a ser estudado, a estratégia a ser empregada e os procedimentos realizados. No entanto, o poder da trajetória hipotética está na ação de o professor vislumbrar os diferentes: caminhos pelos quais os estudantes podem passar; problemas que podem formular; estratégias de ação 
que podem adotar; procedimentos que saibam utilizar. Consideramos que não necessariamente todos os objetivos e hipóteses previstas na THA devam ser obrigatoriamente cumpridos, pois busca-se obter com ela uma maior liberdade e riqueza de planejamento, ao invés de uma "receita" que deva ser seguida.

De posses da proposta de utilizar a THA como recurso no planejamento, do trabalho realizado em um minicurso com estudantes de licenciatura e docentes, das informações coletadas por meio de uma atividade de Modelagem Matemática e da análise realizada, cuja intenção foi de relacionar as produções apresentadas à Trajetória Hipotética de Aprendizagem (THA) antecipadamente planejada, podemos considerar que:

(i) a elaboração de uma THA é uma atividade que pode subsidiar a tomada de decisões docente, pode conferir ao professor segurança ao antecipar em seu planejamento situações possíveis e/ou imprevistas;

(ii) a utilização de uma tarefa de modelagem move a concepção de conhecimento curricular contextualizado; a concepção de aprendizagem matemática dela subjacente; o desenvolvimento de competências associadas ao seu processo; e como consequência natural do processo, a aprendizagem matemática;

(iii) a proposta de utilizar uma $\mathrm{THA}^{10}$ como recurso no planejamento de atividades de Modelagem Matemática pode se apresentar como uma estratégia na formação docente ou continuada, tanto para quem elabora um curso de formação continuada, tanto para quem dele participa.

No que compete especificamente à Trajetória Hipotética de Aprendizagem como instrumento norteador da prática docente podemos destacar sua relevância ao fornecer:

- uma visão sobre os elementos a serem ensinados (I - objetivos);

- os caminhos e recursos didáticos (II - das tarefas de aprendizagem);

- as estratégias metodológicas, condições e hipóteses necessárias (III - processo de aprendizagem).

O movimento de articulação desses três elementos, além de ser próprio da tarefa docente, pode apresentar-se como uma ação de vislumbrar o processo de ensino consonante ao de aprendizagem. A riqueza dessa articulação não reside nos elementos em si, mas sim nos movimentos entre eles, na coerência, no atendimento à concepção de uma prática voltada para a construção de competências, no poder argumentador que as hipóteses podem suscitar.

\footnotetext{
${ }^{10} \mathrm{O}$ potencial da THA, ao nosso ver, não está apenas nas múltiplas atividades que pode propiciar, mas sim nos múltiplos processos de desenvolvimento e estes podem ser derivados de uma única atividade quando se trata de Modelagem Matemática.
} 
Assim, é nesse contexto que a utilização da Modelagem Matemática se insere.

A Modelagem Matemática tem em si uma amplitude de conceitos e atitudes intrínsecas, próprios de sua natureza. Nesse sentido, corroboramos com Caldeira (2015, p. 5960) de que a Modelagem Matemática em sala de aula faz o

\footnotetext{
aluno perceber que determinados conteúdos existem e podem ser aprendidos quando tivermos a oportunidade de percebê-los como instrumentos de compreensão de uma dada realidade, que esteja em interdependência com outros conteúdos que não somente os da matemática por ela mesma.
}

Entendemos que a utilização de Trajetórias Hipotéticas de Aprendizagem coloca em prática não apenas a Modelagem Matemática em ambientes de ensino e aprendizagem, mas quaisquer outras estratégias que valorizem um trabalho docente flexível e dinâmico, voltado para a formação de competências e, consequentemente, de Matemática.

Ainda, diante do que foi evidenciado na investigação, consideramos que o planejamento de um trabalho com Modelagem Matemática por meio de uma THA pode apresentar-se como uma estratégia de formação docente. Pois, tal como uma THA, a Modelagem Matemática na perspectiva da Educação Matemática leva em conta: as particularidades dos projetos individuais de matematização dos estudantes; as possíveis descontinuidades do processo, natural do aprender; a possibilidade de aprender conceitos e conteúdos, implementar atitudes, desenvolver competências; a suposição de que não existe um processo único e neutro de aprendizagem. Considerações estas que são elementos fundamentais e articuladores para um currículo contextualizado na realidade local, social e individual da escola e do seu alunado, configurando-se em uma possibilidade de continuidade de pesquisa.

\section{Referências}

ALMEIDA, L. M. W. ; FERRUZZI, E. C. Uma aproximação socioepistemológica para a modelagem matemática. Alexandria. Revista de Educação em Ciência e Tecnologia, v. 2, n. 2, p. 117-134, 2009.

ALMEIDA, L. M. W.; SILVA, A. Por uma Educação Matemática Crítica: a Modelagem Matemática como alternativa. Educação Matemática Pesquisa, São Paulo, v.12, n.2, p. 221-241, 2010.

ALMEIDA, L. M. W.; SILVA, K. A. P. A Ação dos Signos e o Conhecimento dos Alunos em Atividades de Modelagem Matemática. Bolema, Rio Claro, v. 31, n. 57, p. 202-219, abr. 2017.

ALMEIDA, L. W.; SILVA, K. P.; VERTUAN, R. E. Modelagem Matemática na Educação Básica. São Paulo: Contexto, 2012.

ARAÚJO, J. L.; CAMPOS, I. S. Negotiating the Use of Mathematics in a Mathematical Modelling Project. In: STILLMAN, G. A.; BLUM, W.; BIEMBENGUT, M. S. (Ed.). Mathematical Modelling in Education Research and Practice: cultural, social and cognitive influences. New York: Springer, 2015. p. 283-291. 
BARDIN, L. Análise de conteúdo. 3. ed. Lisboa: Edições 70 Ltda., 2004.

BELTRÃO, M. E. P.; IGLIORI, S. B. C. Modelagem Matemática e Aplicações: Abordagens Para o Ensino de Funções. Educação Matemática Pesquisa, São Paulo, v. 12, n. 1, p.17-42, 2010.

BORROMEO FERRI, R. Theoretical and empirical differentiations of phases in the modeling process. Zentralblatt für Didaktik der Mathematik - ZDM - The International Journal on Mathematics Education, Karlsruhe, v. 38, n. 2, p. 86-95, 2006.

CALDEIRA, A. D. Modelagem Matemática, Currículo e Formação de Professores: Obstáculos e Apontamentos. Educação Matemática Revista, São Paulo, n. 46, p. 53-62, set. 2015.

CARLSON, M. A.; WICKSTROM, M. H.; BURROUGHS, E. A.; FULTON, E. W. A case for Mathematical Modeling in the Elementary School Classroom. In: NCTM. Mathematical Modeling and Modeling Mathematics. Reston: National Council of Teachers of Mathematics, 2016. p. 121129.

DINIZ, L. N.; BORBA, M. C. Leitura e interpretação de dados prontos em um ambiente de modelagem e tecnologias digitais: o mosaico em movimento. Bolema, Rio Claro, v. 26, n. 43, p. 935962, ago. 2012.

GEIGER, V.; ÄRLEBÄCK, J. B.; FREJD, P. Interpreting Curricula to find: opportunities for modeling: case studies from Australia and Sweden. In: NCTM. Mathematical Modeling and Modeling Mathematics Reston: National Council of Teachers of Mathematics, 2016. p. 207-215.

GÓMEZ, P.; GONZÁLEZ, M. J.; LUPIÁÑEZ, J. L. Adapting the Hypothetical Learning Trajectory Notion to Secondary Preservice Teacher Training. Chipre: Universidade de Chipre, 2007.

MELO, T. B.; CHRISPINO, A. Concepção de não neutralidade dos modelos matemáticos: uma experiência no ensino médio. Educação Matemática Pesquisa, São Paulo, v. 15, n. 1, p. 125-146, 2013.

PIRES, C. M. C. Perspectivas construtivistas e organizações curriculares: um encontro com as formulações de Martin Simon. Educação Matemática Pesquisa. São Paulo, v. 11, n. 1, p. 145 - 166, 2009.

SCHROETTER, S. M.; STAHL, N. S.; CHRYSOSTOMO, C. S.; DUNCAN, C. R. A escrita e o pensamento matemático no ambiente virtual utilizando a modelagem matemática: experiência de uma turma de $9^{\circ}$ ano. Educação Matemática Pesquisa, São Paulo, v. 18, n. 1, p. 373-396, 2016.

SILVA, K. A. P. Aspectos cognitivos em aulas com modelagem matemática na disciplina de Cálculo Diferencial e Integral. Experiências em Ensino de Ciências, Cuiabá, v. 12, n. 2, p.156-170, 2017.

SILVA, R. S.; BARONE, D. A. C.; BASSO, M. V. A. Modelagem matemática e tecnologias digitais: uma aprendizagem baseada na ação. Educação Matemática Pesquisa, São Paulo, v. 18, n. 1, p. 421446, 2016.

SIMON, M. A. Reconstructing Mathematics Pedagogy from a Constructivist Perspective. Journal for research in Mathematics Education, Reston, v. 26, n. 2. p.114-145, 1995.

SIMON, M. A.; TZUR, R. Explicating the role of mathematical tasks in conceptual learning: an elaboration of the hypothetical learning trajectory. Mathematical Thinking and Learning, Abingdon, v. 6, n. 2, p. 91-104, 2004. 
STEFFE, L. P. On the Construction of Learning Trajectories of Children: The Case of Commensurate Fractions. Mathematical Thinking and Learning, Abingdon, v. 6, n. 2, p. 129-162, 2004.

STILLMAN, G. A.; BROWN, J. P.; GEIGER, V. Facilitating Mathematisation in Modelling by Beginning Modellers in Secondary School. In: STILLMAN, G. A.; BLUM, W.; BIEMBENGUT, M. S. (Ed.). Mathematical modelling in education research and practice: Cultural, social and cognitive influences. Cham: Springer, 2015. p. 93-104

Submetido em 28 de Novembro de 2017. Aprovado em 31 de Julho de 2019. 